\title{
Design of active feedback for rehabilitation device
}

\author{
Ondrej Liska ${ }^{1 a}$ Marcel More ${ }^{1}$ Dagmar Janacova ${ }^{2,}$ and Vladimir Vasek ${ }^{2}$ \\ ${ }^{1}$ Technical University of Kosice, Faculty of Mechanical Engineering, Department of Automation, Control and Human Machine \\ Interaction, Letná 9, 04200 Košice, Slovakia \\ ${ }^{2}$ Tomas Bata University in Zlin, Faculty of Applied Informatics, nám. T.G.Masaryka 5555, 76001 Zlín. Czech republic
}

\begin{abstract}
Sensor systems are an essential part of automated equipment. They are even more important in machines that come in contact with people, because they have a significant impact on safety. This paper describes the design of active feedback for rehabilitation device driven by pneumatic artificial muscles. Here are presented three methods for measuring the load of the robot. The first is a system composed of Force Sensitive Resistors (FSR) placed in the handle of the device. Two other methods are intended to measure the load of the actuator composed of artificial muscles. The principle of one method is to measure the difference in filling pressures of the muscles, second is based on strain measurement in the drive cables. The paper describes advantages and disadvantages of using each of these methods in a rehabilitation device
\end{abstract}

\section{Introduction}

Musculoskeletal rehabilitation is an essential part of the healing process of patients who have experienced a traumatic injury or stroke limiting their range of motion. But rehabilitation exercises carried out by a physiotherapist are time-consuming and expensive. For these reasons, there is an endeavor to at least partially automate this task with usage of robotic rehabilitation devices. Their advantage is that the patient himself can choose the time and intensity of the exercise and especially in the case of simpler devices it is also possible to use them at the patient's home.

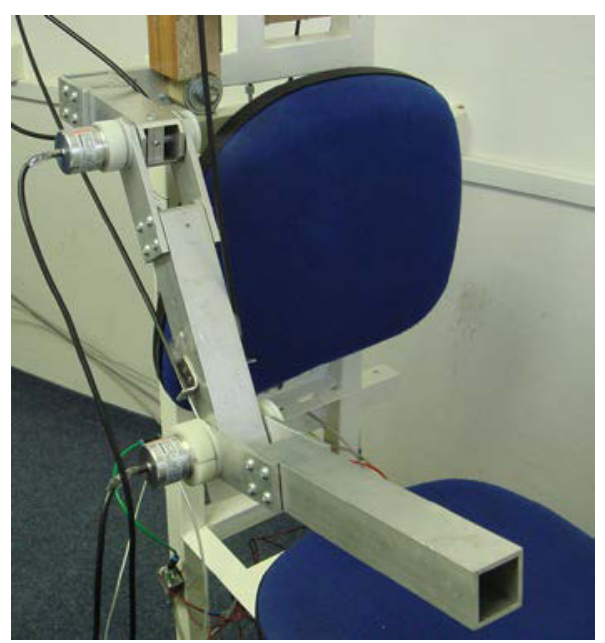

Figure 1. Rehabilitation device driven by pneumatic artificial muscles
In our department we are developing device for rehabilitation of the upper limb joints. It is designed for exercising shoulder and elbow joint at the same time. It's in the form of exoskeleton, so its kinematics reproduces kinematics of the human arm. The main specific of this device is actuator used to drive him - pneumatic artificial muscles.

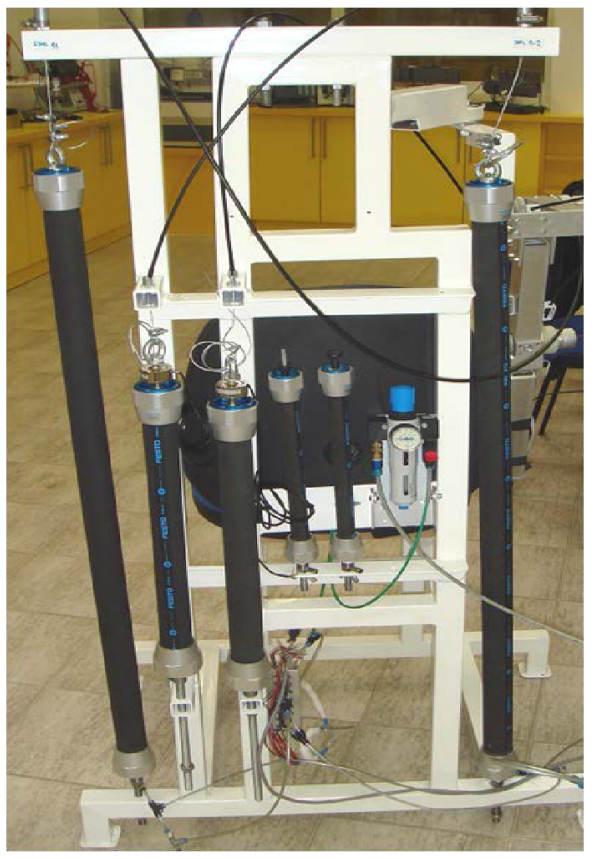

Figure 2. Rehabilitation device driven by pneumatic artificial muscles - rear view

\footnotetext{
$\overline{\mathrm{a}}$ Corresponding author: ondrej.liska@tuke.sk
} 
Device as is shown in Figure 1and 2 can perform passive rehabilitation exercises. For these exercises, it is sufficient that the device have a control loops for position and speed control of the device joints. But at some stage of recovery, it is necessary to start with active exercises in which the patient has to use his own strength. For the automated rehabilitation system, this means that this force should be measured and regulated. However, force measurement is problematic part in the design of sensor system. Since this device is specific by its purpose (rehabilitation) and also because of its drive (pneumatic muscles), there are no ready-made solutions that can be directly applicable. Because measured values should be used for direct control, this method must provide them in real time, without significant delay and without so called time errors.

\section{Analysis of force measurement}

Principled model of robotic arm with one joint driven by a pair of antagonistically connected artificial muscles is shown in Figure 3. On this model there are shows possible placements of force measuring system. In this case, the load is considered to be solely on the end of the robots arm.

The load can be measured on these three places:

1. at the point of contact,

2. at the robots arm - measurement of deformation,

3 . in the actuator - measurement of load.

\subsection{Measuring deformation of the robots arm}

In this method, the force is measured indirectly as a deformation of the robots arm. For small deformation measurement in experimental mechanics but also in practical applications there are used strain gauges and load cells. For measuring the load of rehabilitation robot we considered two methods. The first is application of strain gauges directly to the robots arm. But this includes one difficult task. It is necessary to create the point, in which the deformation will be sufficient for measurement with satisfying resolution, while maintaining the strength of this element. This disadvantage can be eliminated by usage of bending load cell. These sensors mostly consist of a steel body on which the strain gauges connected to Wheatstone bridge are mounted. They are designed to measure strain in their nominal range but they can withstand some overload (typically 150-200\%) [1]. Sensor like this can be embedded in the robots arm. The disadvantage of this solution is its dimensions and weight. Because of the material of load cell (mostly steel), the sensor placed on the end of kinematic chain can create significant torque.

\subsection{Measurement of actuator's load}

Rehabilitation device is designed so that each member of the kinematic chain has one degree of freedom. This is where the actuator operates and also where we need to measure load. In conventional robots that use electric actuators, the load is mostly determined indirectly by measuring the current that passes through the motor. When using a pneumatic actuator this solution can't be used. But in the devices driven by artificial muscles other variables specific to this type of drive can be measured. Application of external forces to such an actuator leads both to change of pressure in both pneumatic muscles and also to change of stress in other components such as cables, chain, pulleys, etc.

\section{Methods selected for further evaluation}

For further consideration, we have selected three measurement methods. We choose these methods based on their advantages but also for their affordability and suitability for incorporation into existing rehabilitation device.

\subsection{Force Sensitive Resistors (FSR)}

FSR are passive electronic components that change their resistance according to applied force. These sensors consist of several thin layers of polymer so that application of force to the active area results in reduction of electrical resistance. Mechanically and also by range of measurement are these sensors designed to measure force of human touch. FSRs are not intended to accurate measurements like strain gauges.
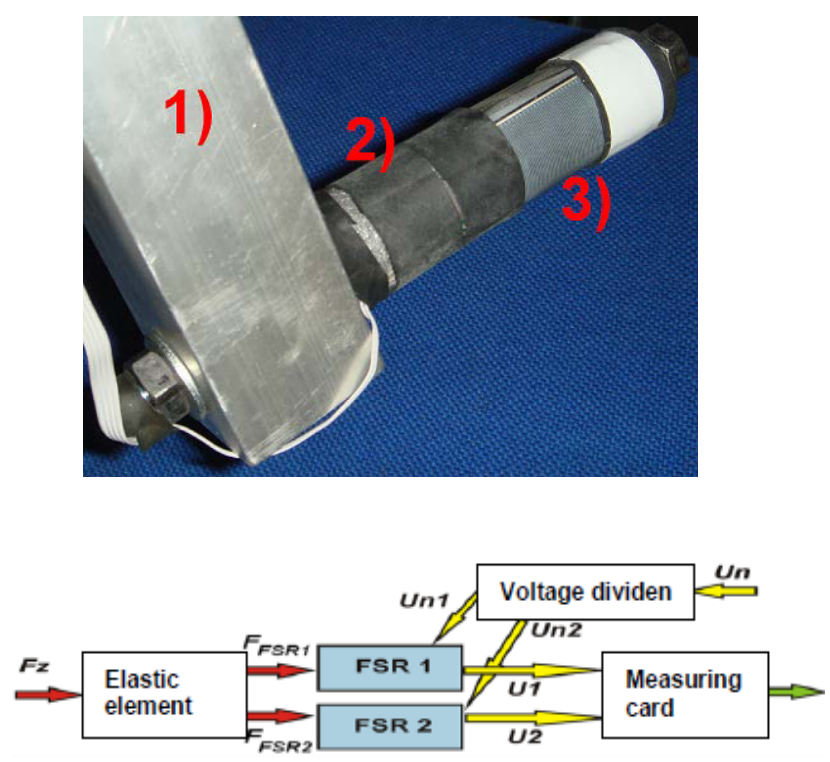

Figure 3. FSR sensors mounted on handle - 1) robots arm, 2) handle, 3) FSR

The force between rehabilitation device and patient is transferred mainly through the handle. This is place where direct contact occurs on relatively small area, which can be covered by conventional sizes of these sensors $\left(0.25\right.$ to $\left.25 \mathrm{~cm}^{2}\right)$. Output characteristic of the sensor (resistance dependence on the strength) is roughly linear, so simple algorithms are sufficient for the signal 
processing. Because this is a simple passive element, this characteristic can be modified by various electrical connections. If necessary, it is also possible to create different types of output - voltage, current, frequency and so [2]. To determine the force in the direction of one axis, it is necessary to know the values measured in opposing sensors $\left(F_{x 1}, F_{x 2}\right)$.

The force on this axis is then the difference of their values $\Delta F_{x}$.
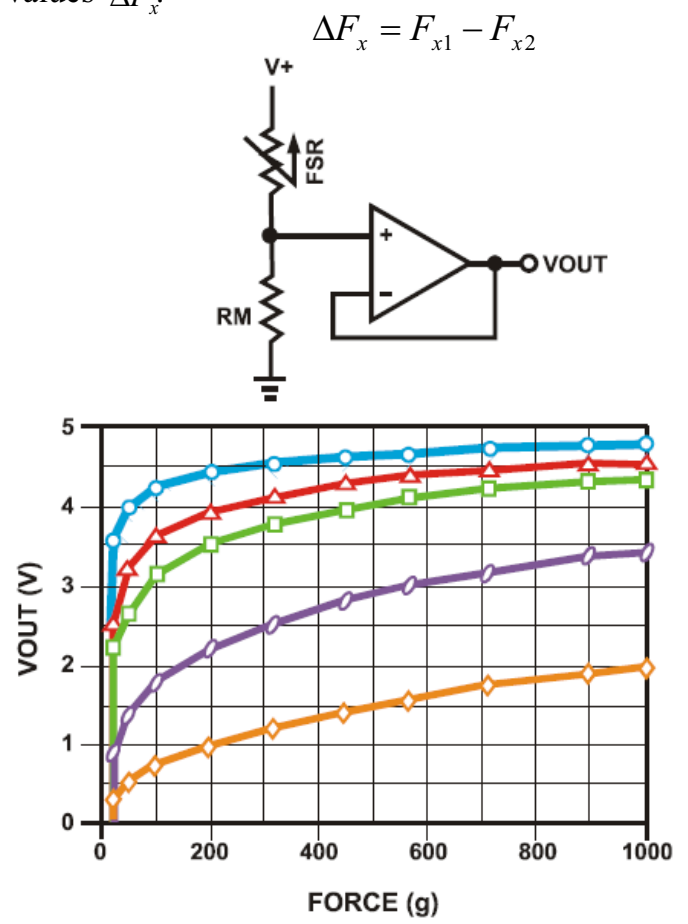

RM VALUES

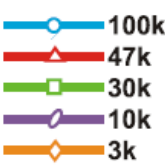

When the force is applied to the muscle, it changes its length. With the change of length it also changes its volume, which in a closed system leads to a change in pressure. This means that we can determine load of the robot by measuring values of pneumatic pressure in muscles. The process of calculating force from pressure is described bellow. First, we must determine value of the nominal difference between the pressures in the muscles $\Delta P_{n}$ at given position (measured by encoder). For this purpose it is necessary to assemble the model or mathematical dependence of pressure on the angle of the joint $\varphi$.

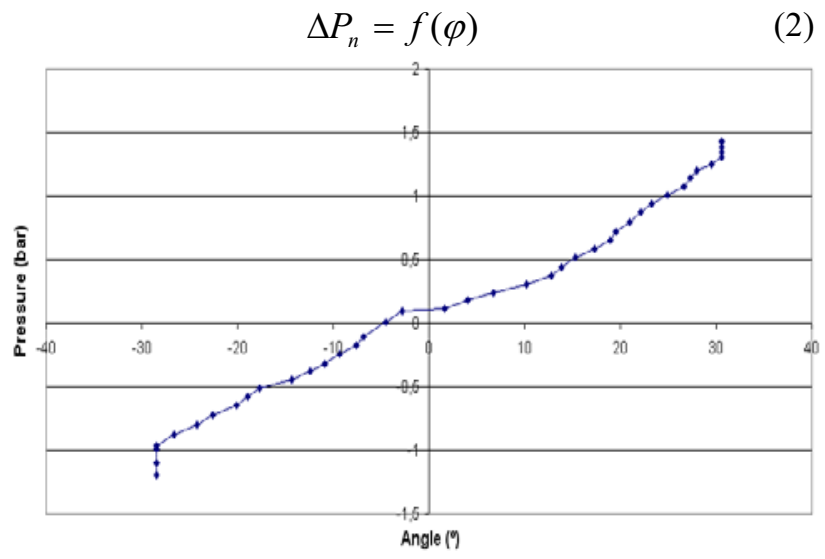

Figure 5. Dependence of pressure difference on angle of the joint

A complication in this step can be a various non-linear properties, which are characteristic for these actuators. However, it has significant impact on accuracy of this method of measurement. The next step is to calculate the actual pressure difference betwee values $\Delta P_{s}$ of pressure in these muscles $\left(P_{1}, P_{2}\right)$.

$$
\Delta P_{s}=P_{1}-P_{2}
$$

Finally we can calculate the torque $(M)$ as the function of difference between real and nominal pressure differences. Because the ratio of transmission is known and constant, we can then calculate the force.

$$
M=f\left(\Delta P_{s}-\Delta P_{n}\right)
$$

Different types of sensors can be used for pressure measurement. It is possible to use differential sensor, which provide directly the difference between two pneumatic pressures. But here, the more useful solution is to use single ended sensor for each muscle so it can be used also for different tasks (like in algorithm, which prevents complete exhaust of muscle). The disadvantage of this measuring method is that the measured values can be affected by pressure fluctuations caused by pneumatic valves during operation. This phenomenon is less significant when we use control methods that change the pressure in muscles smoothly (proportional valves, etc.). Another optimization can be done by mounting sensor in proper location. Since the pressure fluctuations are largest near the valve, pressure sensor should be connected as close to the muscle as possible (Fig. 6 left). However,

\subsection{Measuring actuator's load based on pneumatic pressure}

) 
best location is on the other side of the muscle, opposite to the opening where the valve is connected (Fig. 6 right). But for this, it is necessary to use muscle that is opened on booth sides.

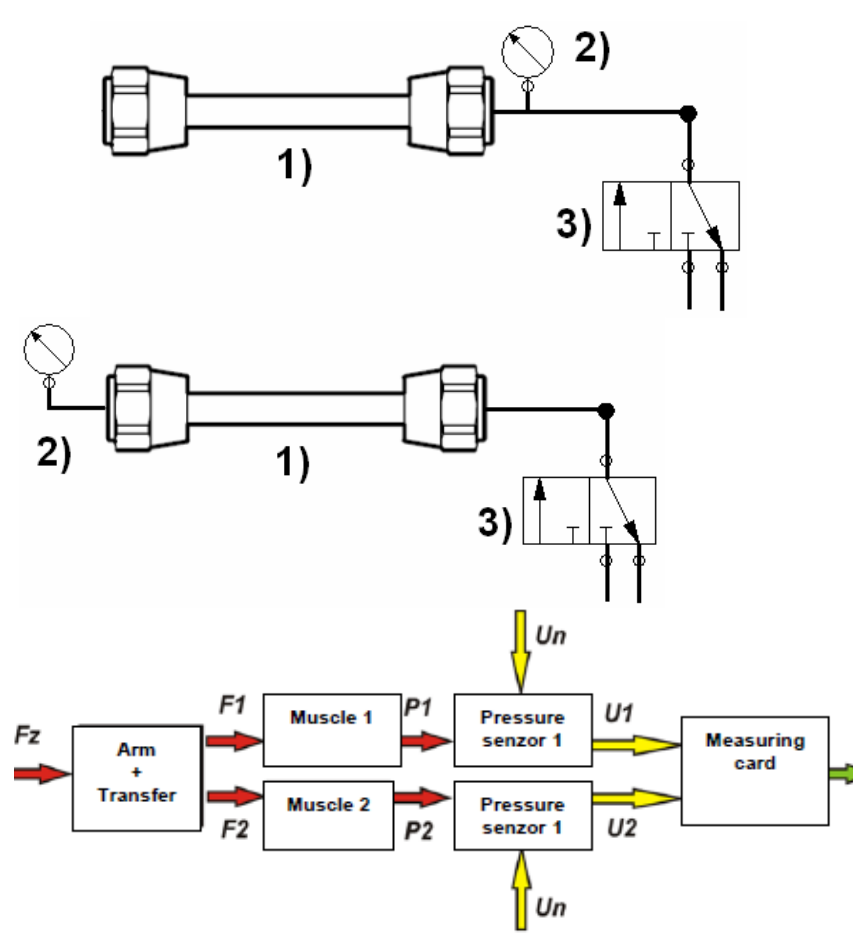

Figure 6. Options for placement of pressure sensor on muscle opened on one (up) and booth ends (down)

\subsection{Measuring actuator's load based on strain in cables}

Strain in the cables can be measured by load cells. Sensors for measuring tensile forces are small in size and relatively light and thus can be easily incorporated into the system. On Fig. 7 it is possible to see an example of their placement between pneumatic muscle and cable. Dimensions of this sensor are $\varnothing 34 \times 18 \mathrm{~mm}$, so it does not increase the outline of a muscle in diameter and only slightly increases its length. Since booth muscle and cable transfer force only in one axis, sensor can be loaded only by tensile stress. Although the sensor is placed on the moving component, the movement of the muscle is in the order of centimeter, so the supply and signal conductors don't limit its use. Also measurement apparatus can be placed relatively close to the sensors. Processing of measured values is similar to the previous case. For the determination of the force it is necessary to calculate the difference $\Delta F$ between forces in each cable $\left(F_{1}, F_{2}\right)$.

$$
\Delta F=F_{1}-F_{2}
$$

Even if the forces measured on unloaded arm should be theoretically balanced, in the practical implementation it is necessary to consider the effect of gravity. This affects the robots arm differently depending on its position and also on the position of other members of the kinematic chain.

Therefore, as in the previous case, it is necessary to determine a dependence of the force $\left(F_{G}\right)$ on the position of the $\operatorname{arm}(\varphi)$.

$$
F_{G}=f(\varphi)
$$

Then the effect of this force is subtracted from the measured values to get the real value of force exerted by the patient $\left(F_{P}\right)$.

$$
F_{P}=\Delta F-F_{G}
$$
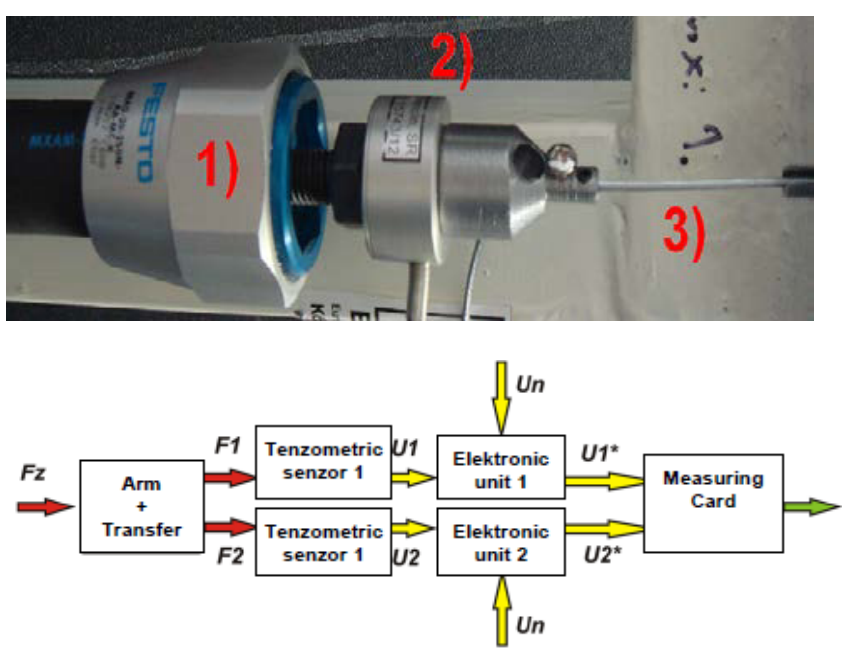

Figure 7. Load cell for measuring tensile forces - 1) muscle, 2) load cell, 3) cable

\section{Conclusion}

Force feedback is essential for the automated device that is intended for active rehabilitation. But in the device driven by pneumatic artificial muscles, we can not use the force measuring system used in conventional robots. It is therefore necessary to assemble a measuring system which takes into account his specific characteristics and requirements. Analysis of the available options shows that load can be measured in three locations: at the point of contact, at the robot's arm or in the drive. For closer comparison we selected three methods. The first is measurement in the handle of the device using FSR sensors. Other two methods use the fact that the force applied to the robot arm creates appropriate response in the drive. In case of actuator composed of pneumatic muscles, this load can be determined from the change in filling pressure in each muscle. Because the pressure sensors are a standard part of systems containing pneumatic circuits, such measurement requires no additional hardware components. Application of the load also results in the strain in mechanical parts of the drive and can be measured by load cells. Although this method is relatively complex, it gives results least influenced by other processes and random errors. These three methods will be evaluated in our department to determine the most 
suitable method for measuring force in unconventional rehabilitation device.

\section{Acknowledgement}

The research work is supported by the Project of the Structural Funds of the EU, Operational Programme Research and Development, Measure 2.2 Transfer of knowledge and technology from research and development into practice. Title of the project: „Research and development of intelligent nonconventional actuators based on artificial muscles", ITMS code: 26220220103, by the Slovak Grant Agency VEGA contract $\mathrm{Nb}$. 1/0911/14 "Implementation of wireless technologies into the design of new products and services to protect human health" and by the Ministry of Education, Youth and Sports of the Czech Republic within the National Sustainability Programme project No. LO1303 (MSMT7778/2014) and also by the European Regional Development Fund under the project CEBIA-Tech No. CZ.1.05/2.1.00/03.0089

\section{References}

1. Emsyst Shear-web Load Cell EMS30. Avalibe:http://emsyst.sk/images/snimace_sily/standa rtne/EMS30a.pdf

2. Interlink Electronics: FSR Force Sensing Resistor Integration Guide and Evalution Parts Catalog. Available: http://www.imagesco.com/sensors/fsr/

3. A. Erwin et al.: Interaction Control For Rehabilitation Robotics via a Low-Cost Force Sensing Handle, in: DSCC2013 Proceedings of the ASME 2013 Dynamic Systems and Control Conference Available: http://mahilab.rice.edu/sites. (2013)

4. F. Trebuna, P. Sivak, Experimentálne metódy mechaniky: Tenzometria, Košice : TU, ISBN 97880-553-1378-8. (2012)

5. A. Hosovsky et al.: Model-based Evolution of a Fast Hybrid Fuzzy Adaptive Controller for a Pneumatic Muscle Actuator, in: International Journal of Advanced Robotic Systems. Vol. 9 (56). pp. 1-11. ISSN 1729-8806. (2012)

6. I. Mack et al.: Interactive Force Sensing Feedback System for Remote Robotic Laparoscopic Surgery, in: Haptic Audio visual Environments and Games. (2009) Available: http://ieeexplore.ieee.org/stamp

7. T. Kelemenova et al:: Model based design and HIL simulations, in: American Journal of Mechanical Engineering. Vol. 1 pp. 276-28. ISSN 2328-4102. (2013)

8. K. Zidek, A. Hosovsky: Wireless Device Based on MEMS Sensors and Bluetooth Low Energy (LESmart) Technology for Diagnostics of Mechatronic Systems, In: Applied Mechanics and Materials. Vol. 460, pp. 13-21. ISSN 1660-9336. (2014)
9. L. Vasek, V. Dolinay Simulation model of heat distribution and consumption in municipal heating network, International Journal of Mathematical Models and Methods in Applied Sciences, Volume 4, 4, pages 240-248 (2010)

10. P. Berkelman et al.: A Miniature Microsurgical Instrument Tip Force Sensor for Enhanced Force Feedback During Robot-Assisted Manipulation, In: IEEE Transactions On Robotics And Automation. (2003)

11. M. Tothova, J. Pitel: Dynamic model of pneumatic actuator based on advanced geometric muscle model, in ICCC 2013: IEEE 9th International Conference on Computational Cybernetics, IEEE pp. 83-87. ISBN 978-1-4799-0060-2. (2013)

12. M. Dalvand et al.: Force Measurement Capability for Robotic Assisted Minimally Invasive Surgery Systems, in: Proceedings of the World Congress on Engineering and Computer Science. (2013) 